\title{
Fabrication and geometric characterization of highly-ordered hexagonally arranged arrays of nanoporous anodic alumina
}

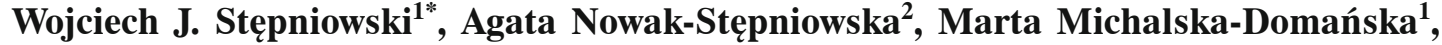 \\ Małgorzata Norek', Tomasz Czujko', Zbigniew Bojar ${ }^{1}$ \\ ${ }^{1}$ Military University of Technology, Department of Advanced Materials and Technologies, Faculty of Advanced Technologies \\ and Chemistry, Kaliskiego 2 Str., 00-908 Warszawa, Poland \\ ${ }^{2}$ Military University of Technology, Institute of Optoelectronics, Kaliskiego 2 Str., 00-908 Warszawa, Poland \\ *Corresponding author: e-mail: wstepniowski@wat.edu.pl
}

\begin{abstract}
Anodic aluminum oxide (AAO) has been fabricated in the $0.3 \mathrm{M}$ oxalic acid at voltage range $20-60 \mathrm{~V}$ and temperature range of $35-50^{\circ} \mathrm{C}$. The resulting nanoporous alumina surfaces were characterized by high resolution scanning electron microscopy, and the images were quantitatively analysed by means of an innovative approach based on fast Fourier transform. The influence of operating anodization voltage and electrolyte temperature on nanopores geometry (pore diameter, interpore distance, porosity, pores density) and arrangement has been studied in details and compared to literature data and theoretical calculations. It was found that independently from the temperature, the best arrangement of the nanopores is for anodic aluminum oxide formed at voltages ranging from 40 to $50 \mathrm{~V}$. Moreover, it was found that pore diameter and interpore distance increase linearly with voltage, what is in line with the literature data.
\end{abstract}

Keywords: nanopores, anodization, self-organization, fast fourier transform.

\section{INTRODUCTION}

Anodic aluminum oxide (AAO) is a nanostructured material formed with electrochemical oxidation of aluminum foil. It consists of parallel, hexagonally arranged nanopores ${ }^{1}$. Nanoporous AAO serves very often as a template for nanofabrication and can be used in numerous applications such as: synthesis of nanowires ${ }^{\mathbf{1 - 1 0}}$, nanotubes $^{15-19}$ and nanodots ${ }^{20}$, magnetic ${ }^{21-23}$ and thermoelectric ${ }^{24}$ materials processing, renewable energy harvesting and storage $^{13,18}$, surface enhanced Raman spectroscopy ${ }^{25-26}$, sensor assembly ${ }^{27-28}$, biocompatible surfaces for living cells integration ${ }^{29}$ and drug delivery systems ${ }^{30-31}$.

Anodization of aluminum is mainly performed in three electrolytes: sulfuric acid, oxalic acid and phosphoric acid at voltage ranges: $15-25^{1,32-33}, 5-100^{1,32,33-36}$ and $150-195^{1,32,37} \mathrm{~V}$ respectively. In order to further expand the anodization voltage ranges, alternative organic acids have also been explored by several authors. Electrolytes like citric ${ }^{38}$, malonic ${ }^{38-39}$, maleic $^{38}$, malic ${ }^{39-40}$, tartaric ${ }^{38-39}$, tartronic ${ }^{39}$, glutaric ${ }^{39}$, lactic $^{41}$, propionic ${ }^{41}$, glycolic ${ }^{41}$ and succinic $^{39,41}$ acids were used.

It is a well-recognized fact that geometrical features of the anodic alumina like: pore diameter, interpore distance, porosity, pores density and thickness of the porous oxide layer, equal to the pores depth, are strictly related to the operating conditions, including: type, concentration and temperature of the electrolyte, applied voltage and duration of the second step of self-organized anodization ${ }^{1,32-38}$. For example, pore diameter and interpore distance increase linearly with increasing volta$\mathrm{ge}^{\mathbf{1}, 32-38}$. Temperature and time increase pore diameter, due to the enhanced etching of the grown oxide by the electrolyte, however neither temperature nor time effect was reported for the interpore distance ${ }^{34}$.

Due to a large variety of applications of anodic alumina, a fast and efficient method of AAO fabrication is demanded. In this paper, fabrication of anodic aluminum oxide (AAO) via 15-minutes long anodizations in $0.3 \mathrm{M}$ oxalic acid at elevated temperatures is investigated, while typical anodization procedures are few hours long, making fabrication of alumina templates time consuming. In this work, the influence of operating conditions on geometrical features (pore diameter, interpore distance, porosity, pores density) and nanopores arrangement with the use of Fast Fourier Transforms (FFT) has been studied in details and compared with the literature data.

\section{EXPERIMENTAL}

Anodic Aluminum Oxide (AAO) was formed according to the previously reported procedure ${ }^{33}$. Briefly, $0.25 \mathrm{~mm}$ thick high-purity aluminum foil $(99.9995 \%$ Puratronic, Alfa-Aesar) was cut into samples with one-side surface area of $25 \mathrm{~mm} \times 5 \mathrm{~mm}$, degreased in acetone and ethanol and electropolished $\left(\mathrm{C}_{2} \mathrm{H}_{5} \mathrm{OH}: \mathrm{HClO}_{4}\right.$ in ratio of $4: 1$ by volume, $0.5 \mathrm{~A} / \mathrm{cm}^{2}, 10^{\circ} \mathrm{C}, 60 \mathrm{~s}$, Pt grid as a cathode). Next, the samples were painted with acid resistant paint (Jedynka ${ }^{\circledR} / 65$ Tikkurila Polska S.A.) at back and edges. Due to the painting, an exposure area of the electropolished aluminum was $50 \mathrm{~mm}^{2}$. The anodizations were performed employing a two-step self-organized procedure. All the anodizations were conducted in $0.3 \mathrm{M}$ oxalic acid, when the first and the second step of anodization was 15 minutes long. The investigated voltage range was 20.0 to $60.0 \mathrm{~V}$ with a step of $5.0 \mathrm{~V}$. The anodizations were performed at four electrolyte temperatures: 35,40 , $45,50^{\circ} \mathrm{C}$. After the first step of anodization, the grown oxide was chemically removed in a mixture of $6 \mathrm{wt} \%$ $\mathrm{H}_{3} \mathrm{PO}_{4}$ and $1.8 \mathrm{wt} \% \mathrm{H}_{2} \mathrm{CrO}_{4}$ at $60^{\circ} \mathrm{C}$. The 90 minutes long chemical etching, the samples were rinsed with deionized water several times and the re-anodizations were performed for the samples at the same experimental conditions like during the first step of anodization.

The morphology of the obtained nanostructures was examined by field-emission scanning electron microscope (FE-SEM) Leo 1530 (Carl Zeiss, Germany). Geometrical features, excluding interpore distance, of the AAO nanostructures were estimated from six independent FE-SEM images of sample obtained at certain set of the 
experimental conditions, with the use of NIS-Elements image analysis software. Fast Fourier Transforms (FFT) and interpore distances were estimated from six independent FE-SEM images at a certain set of the experimental conditions with the use of WSxM software ${ }^{\mathbf{4 2 - 4 3}}$.

\section{RESULTS AND DISCUSSION}

Figure 1 shows FE-SEM images (A, D, G, J) their fast Fourier transforms $(\mathrm{B}, \mathrm{E}, \mathrm{H}, \mathrm{K})$ with radial averages (C, F, I, L) of AAO formed in $0.3 \mathrm{M}$ oxalic acid via 15-minutes long anodizations at $45^{\circ} \mathrm{C}$ at 20.0 (A-C), 30.0 (D-F), 40.0 (G-I) and $50.0 \mathrm{~V}$ (J-L). It can be immediately noticed that voltage increase results in pore diameter and interpore distance increase. Moreover, changes in the FFT images are also noticed: for lower
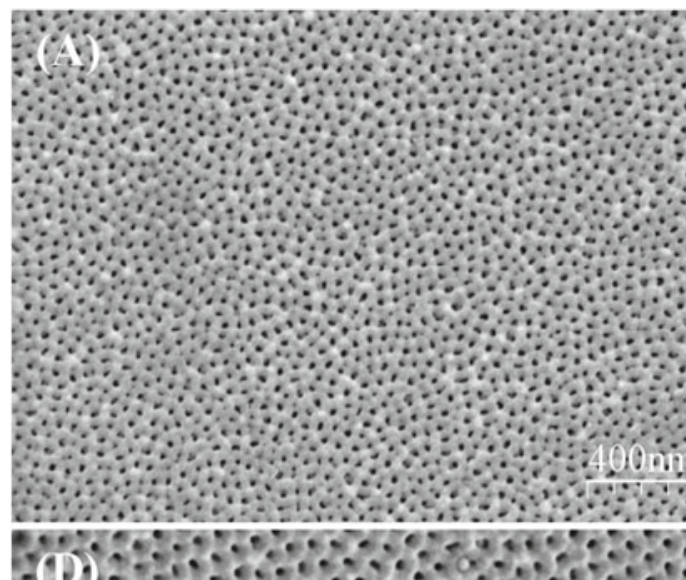

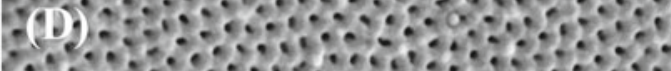

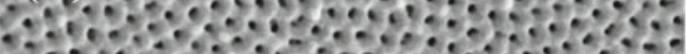

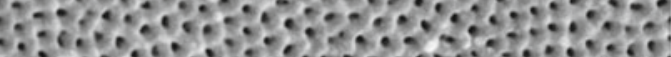

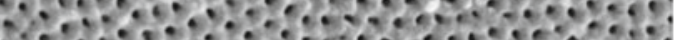

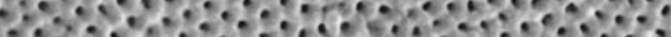

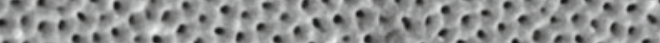

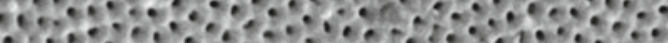

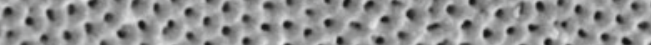

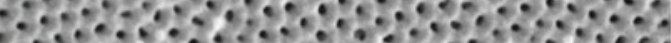

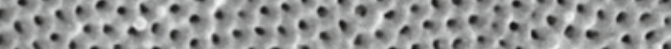

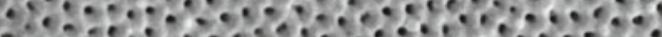

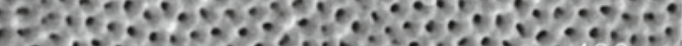

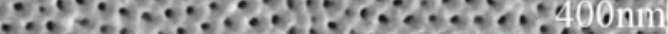

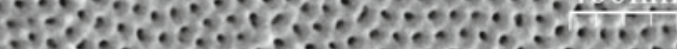

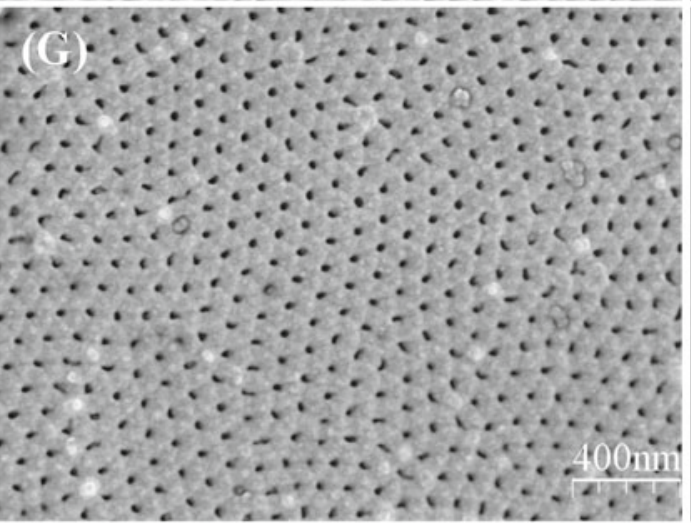
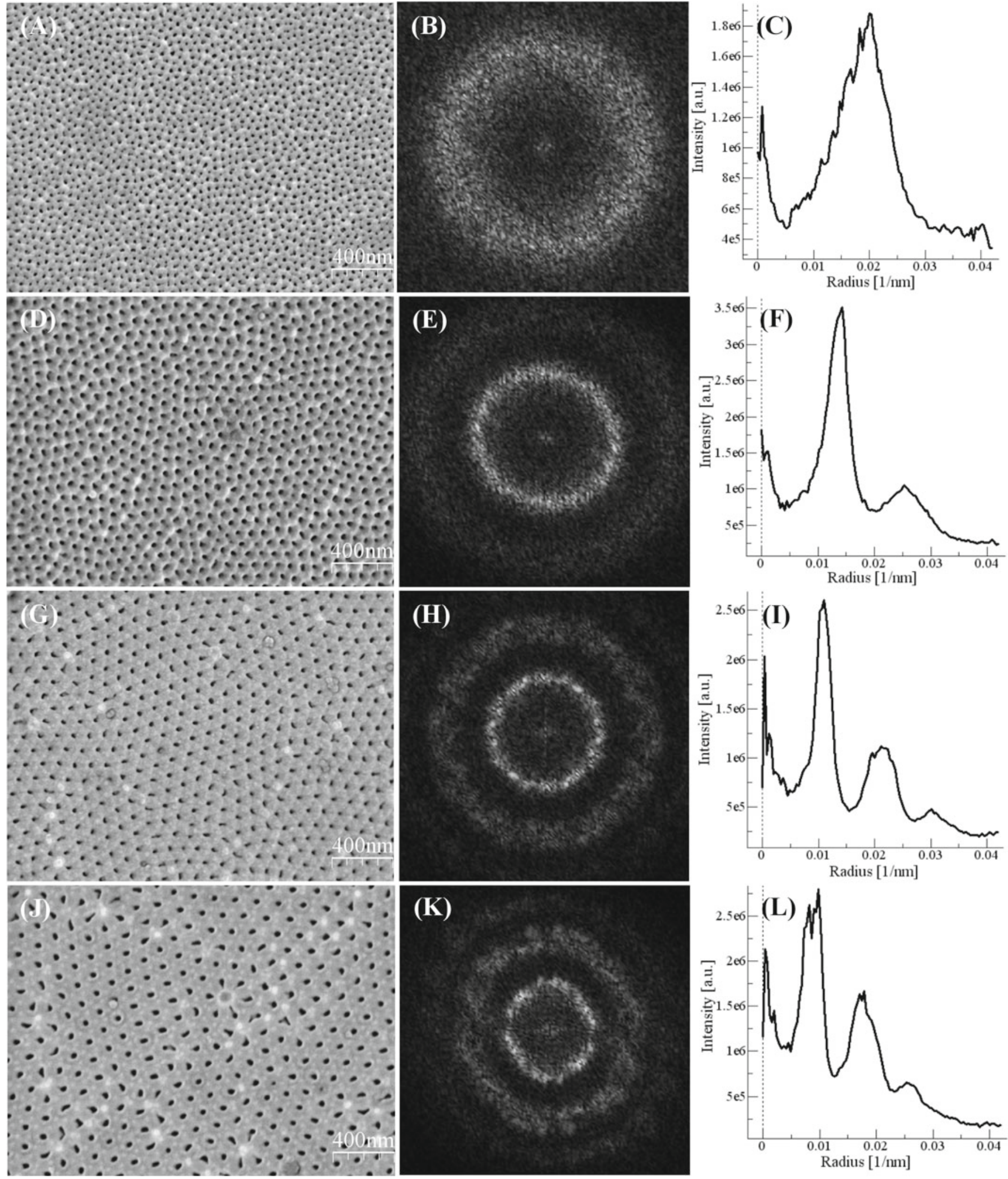

Figure 1. FE-SEM images (A, D, G, J), their fast Fourier transforms (B, E, H, K) with radial averages (C, F, I, L) of anodic aluminum oxide formed in $0.3 \mathrm{M}$ oxalic acid via 15 -minutes long anodizations at $45^{\circ} \mathrm{C}$ at 20.0 (A-C), 30.0 (D-F), 40.0 $(\mathrm{G}-\mathrm{I})$ and $50.0 \mathrm{~V}(\mathrm{~J}-\mathrm{L})$ 
voltages the FFT images are blurred and for 40.0 and 50.0 distinct dots in the corners of the hexagon are seen, what corresponds to the poor and high quality arrangement respectively. Moreover, the radial averages of the FFT also bring the information about the formed AAO nanostructures. The abscissa of the radial average maximum is the inverted value of the average interpore distance at the analyzed image. In Figure 1, shift of the abscissa of the radial average towards lower values with the increasing voltage is observed, what translates into interpore distance increase with the voltage increase, what is in line with the literature data ${ }^{32-34,44}$. Thus, the whole radial average is the interpore distance distribution function. On the basis of the FFT radial average the uniformity of the interpore distances can be quantified, what will be discussed in details in the further text of the paper.

On Figure 2 FE-SEM images (A, D, G, J) and their FFT (B, E, H, K) with radial averages $(\mathrm{C}, \mathrm{F}, \mathrm{I}, \mathrm{L})$ of AAO formed in $0.3 \mathrm{M}$ oxalic acid via 15 -minutes long anodizations at $25.0 \mathrm{~V}$ at 35 (A-C), 40 (D-F), 45 (G-I) and $50^{\circ} \mathrm{C}(\mathrm{J}-\mathrm{L})$ are shown. The pore diameter increase with electrolyte's temperature is noticeable. However, no temperature effect on the interpore distance is observed (no shift of the abscissa of FFT radial average). The ordinate of the FFT changes in radial average maximum position, however no distinct tendency has been observed.

Quantitative analysis of the FE-SEM images shows that pore diameter increases linearly with the voltage (Fig. 3A). It is in accordance with the literature data ${ }^{1 \text {, }}$ $32-35,44$. On the other hand, pore diameter increase with the electrolyte temperature is almost insignificant, probably due to the relatively short time of the anodization. For longer anodizations, pore diameter increase with the electrolyte temperature is much stronger ${ }^{34-35}$. It may be linked with the chemical reaction inside the pores between the grown oxide and the acidic electrolyte. During 15-minutes long anodization, a much smaller quantity of the oxide could react with oxalic acid than during the a few times longer anodizations ${ }^{34}$. According to Pashchanka and Schneider, the temperature of the electrolyte influences two phenomena responsible for the anodic oxide growth: attraction and diffusion ${ }^{46}$. One has to be aware of the complexity of the phenomena at the electrode-electrolyte interface during the anodic oxide growth. A celled anodic oxide growth is a result of Rayleigh-Bénard convection phenomena, where physical quantities like: voltage, anions' charges, electric conductivity and dynamic viscosity of the electrolyte play major roles ${ }^{39,46}$. Moreover, during anodic oxide growth, phenomena like coulombic attraction and diffusion are accompanied by local $\mathrm{pH}$ drops and increases (due to the locally occurring chemical reactions), formation of colloidal oxide-hydroxide $\mathrm{Al}(\mathrm{O}) \mathrm{OH}$ particles and migration of ions ${ }^{39,46}$. thus the statement about the increased chemical reaction rate between electrolyte and grown oxide is just a convenient simplification, useful for the technological purposes.

As it was noticed on the basis of the FE-SEM images and FFT radial averages, interpore distance increases linearly with the voltage (Fig. 1B). The same observations were reported for AAO formed at different operating conditions ${ }^{34-35}$. Moreover, no temperature effect is observed for interpore distances, what is also in line with the literature data ${ }^{34-35,44}$. Additionally, according to theory ${ }^{34-35}$, every voltage increase for $1.0 \mathrm{~V}$ causes interpore distance increase for $2.5 \mathrm{~nm}$ and the fitted curves to the interpore distance - voltage have slope close to the 2.5 (Fig. 3B).

Another quantity characterizing the anodic alumina is porosity. It can be estimated from the pore diameter and interpore distance with the following equation (1) ${ }^{34}$ :

$\alpha=0,907 \cdot\left(\frac{D_{p}}{D_{c}}\right)^{2}$

where a is porosity, $\mathrm{D}_{\mathrm{p}}$ is the pore diameter and $\mathrm{D}_{\mathrm{c}}$ is interpore distance. As it can be concluded from the equation, the porosity should be a quadratic function of the voltage. Porosity estimated from the equation with the use of the experimental data, actually is the quadratic function of the voltage (Fig. 4A). Moreover, it can be seen that the greater the electrolyte's temperature, the greater the porosity, which is caused by increased pore diameter at fixed interpore distance with temperature increase. The greater the porosity, the greater the close-packaging of the deposited individuals while the obtained AAO would be applied as a template for the nanofabrication. Another quantity important for the nanofabrication with the use of AAO is the number of pores occupying a given area, called the pore density. As it can be directly seen from the FE-SEM images, the greater the voltage, the less pores on the given area (Fig. 1). Here, there is also no temperature effect (Fig. 2), because pores density is strictly linked to the interpore distance ${ }^{34}(2)$ :

$n=\frac{10^{6}}{\sqrt{3} \cdot D_{c}^{2}}$

where $\mathrm{n}$ is a number of pores occupying $1 \mathrm{~mm}^{2}$ and $\mathrm{D}_{\mathrm{c}}$ is the interpore distance. In the figure $4 \mathrm{~B}$ one can see a distinct decrease of pores density with the voltage, which is very close, for all the temperatures, to the theoretical equation obtained via substitution of $D_{c}$ by $2.5 \cdot 10^{-3} \mathrm{U}$, where $\mathrm{U}$ is voltage. Therefore, for the smallest voltages, even 350 pores are on $1 \mathrm{~mm}^{2}$. for the smallest voltages, results obtained directly from FE-SEM images and calculated from the theoretical equations are not in a good agreement, because the equation is dedicated to the ideally, hexagonally arranged arrays and the small voltages of anodization spoils the arrangement of the pores.

As it was previously stated, fast Fourier transforms radial average is a distribution function of the inverted values of the interpore distance. Thus, FFT based quantitative arrangement analysis can be done here with the use of the radial averages. It is clear, that the less intensive and wider radial average maximum peak is, the broader is the interpore distance distribution, which means less uniform nanopore arrangement. On the contrary, the more intensity, more distinct and narrower FFT radial average, the better arrangement of the formed anodic aluminum oxide. Therefore, the intensity to the width at half of the peaks height should be a quantity which can reliably describe the interpore distance distribution and the nanopores arrangement in the consequence (3): 

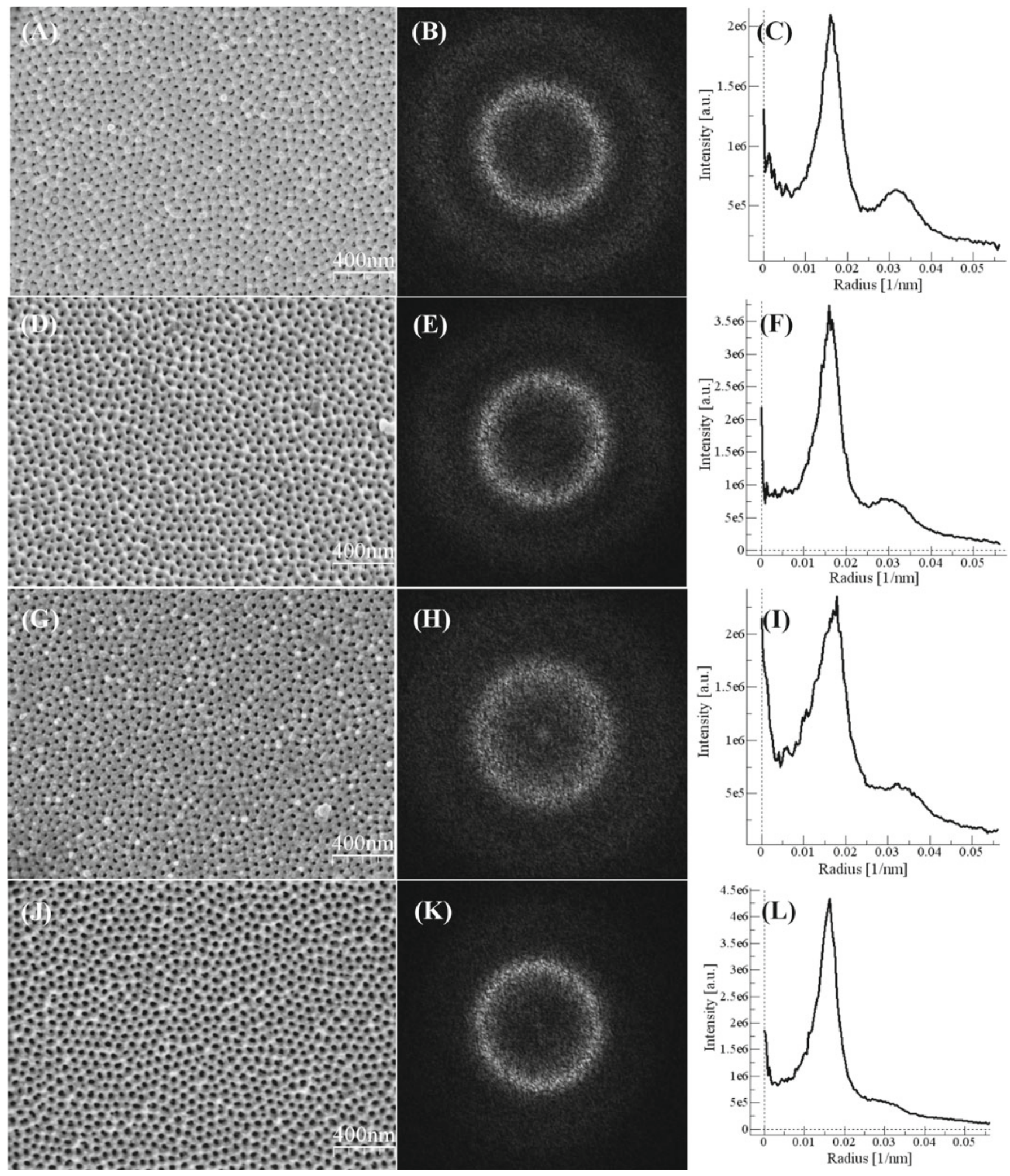

Figure 2. FE-SEM images (A, D, G, J), their fast Fourier transforms (B, E, H, K) with radial averages (C, F, I, L) of anodic aluminum oxide formed in $0.3 \mathrm{M}$ oxalic acid via 15-minutes long anodizations at $25.0 \mathrm{~V}$ at 35 (A-C), 40 (D-F), 45 (G-I) and $50^{\circ} \mathrm{C}(\mathrm{J}-\mathrm{L})$

$R=\frac{I}{W_{1 / 2}}$

where: $\mathrm{R}$ - regularity ratio, $\mathrm{I}$ - intensity, $\mathrm{W}_{1 / 2}$ - width at half of the intensity. Reported approach differs significantly from the previously reported ${ }^{\mathbf{3 6}}$. Typically, FFT derived regularity ratio is based on three independent intensity profiles conducted through the FFT. Here, radial average takes into account the whole FFT what makes the obtained regularity ratio rather more similar to the pair distribution function ${ }^{45}$ than to the classical FFT-based regularity ratio. The quantitative arrangement analysis shows that the best arrangement of the anodic alumina formed via 15-minutes long anodizations in $0.3 \mathrm{M}$ oxalic acid is for nanostructures formed at voltages ranging from 40.0 to $50.0 \mathrm{~V}$, independently on the temperature (Fig. 5). Thus, the results are in a good agreement with the reported analyses conducted with the use of other methods for AAO formed in $0.3 \mathrm{M}$ oxalic acid at lower temperatures and under longer anodization times ${ }^{\mathbf{4 5}}$. 

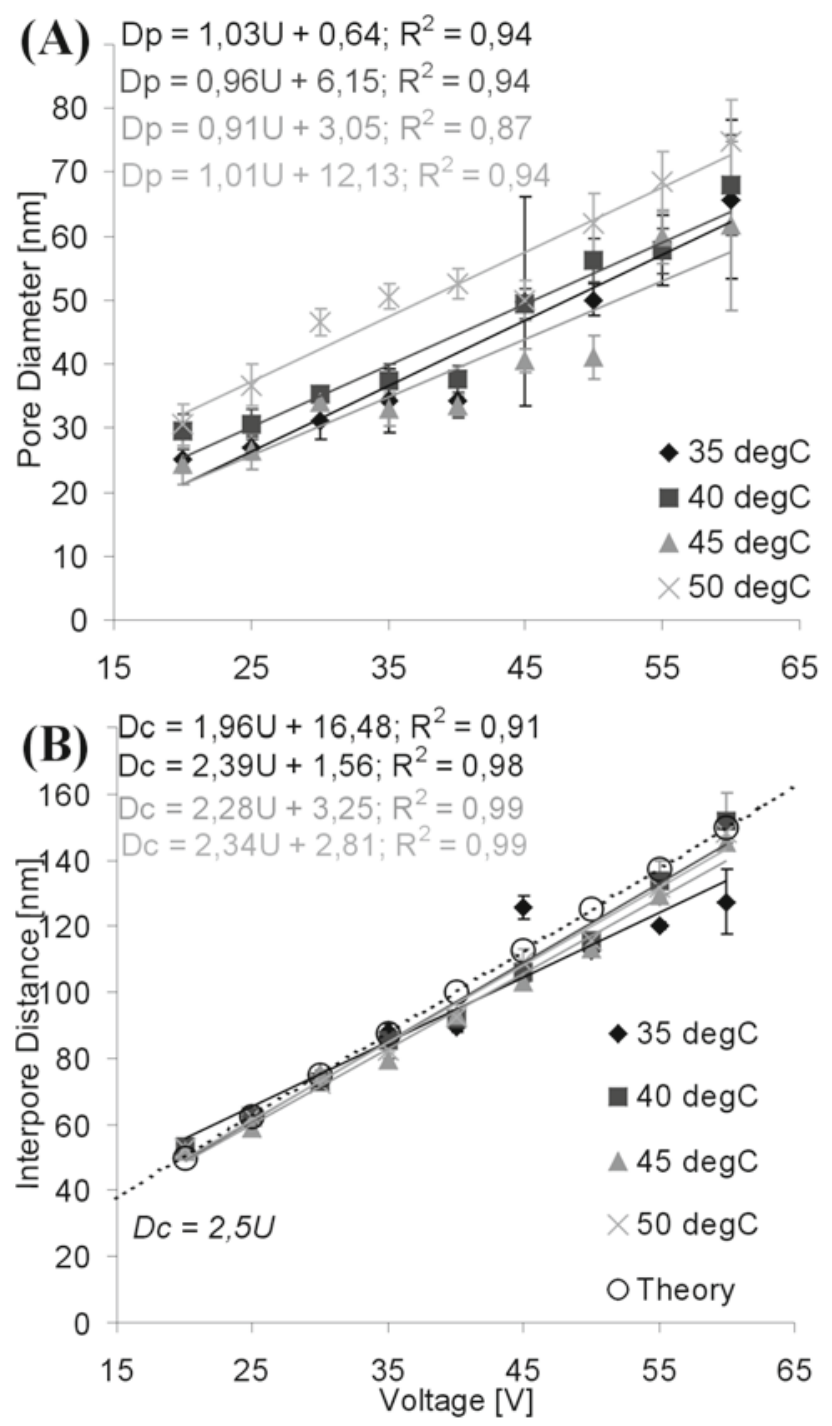

Figure 3. Pore diameter (A) and Interpore distance (B) versus anodizing voltage for various electrolyte's temperatures

Thus the obtained results are in the agreement with the concept reported by Nielsch et al. ${ }^{46}$ over a decade ago, where spatial expansion of the grown oxide is the major factor responsible for the nanopores arrangement, what translates into the best $\mathrm{AAO}$ arrangement at 40 $\mathrm{V}^{47}$. Additionally, the method reported in this paper is more convenient and faster than the typical FFT based regularity ratio, or the preparation of the defect maps.

\section{CONCLUSIONS}

Two step self-organized 15-minutes long anodization in $0.3 \mathrm{M}$ oxalic acid provides anodic aluminum oxide with controllable geometrical features, including pore diameter, interpore distance, porosity and pores density. It was found that pore diameter and interpore distance increase linearly with voltage, what is in agreement with literature data. However, temperature effect on the pore diameter was much smaller than in the case of AAO formed by longer anodizations, even at much smaller temperatures. On the other hand, on increasing the temperature the porosity is increased, what translates into better close-packaging of the pores. Moreover, pores density relies only on the voltage and is in a good agreement with the
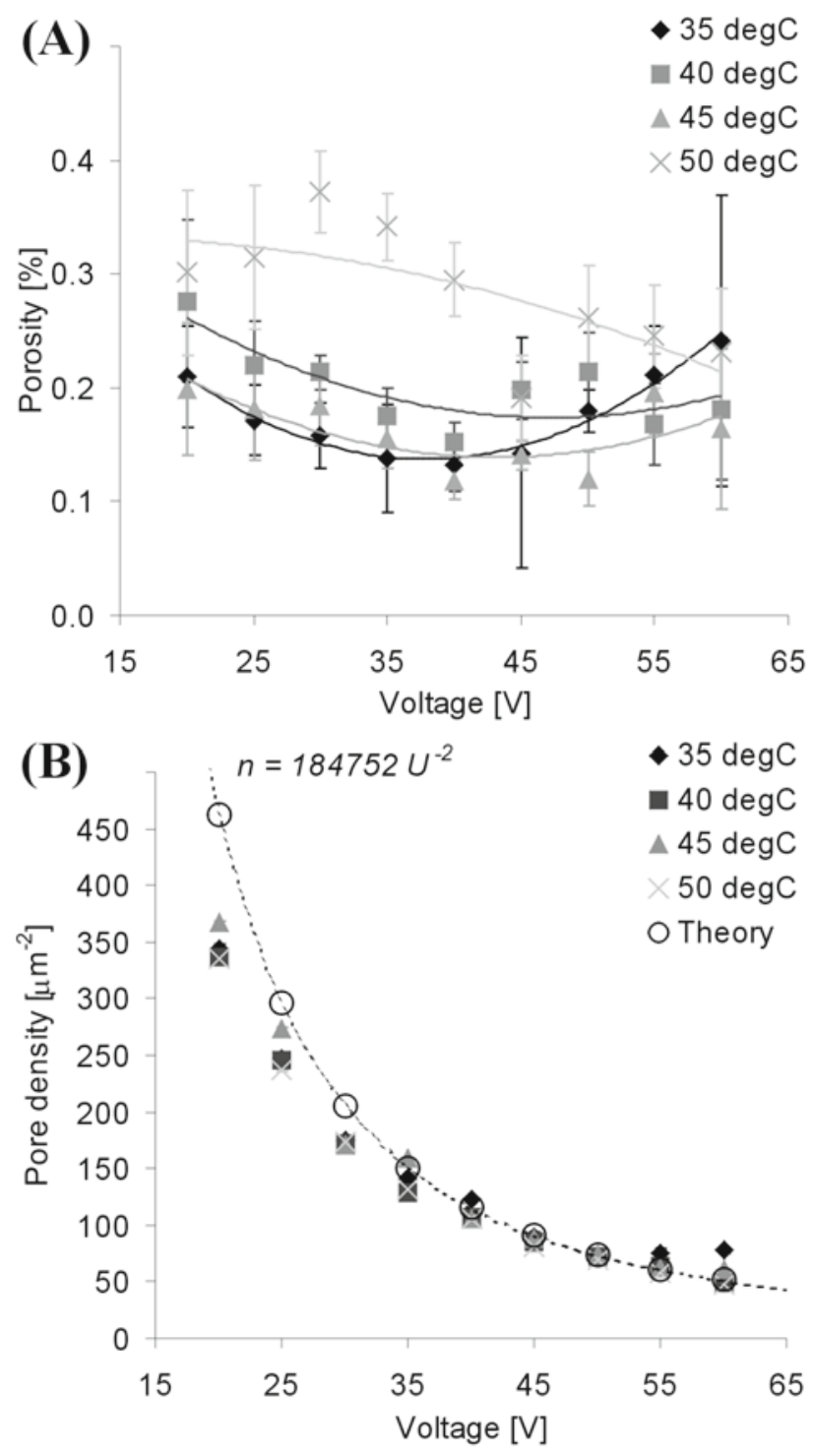

Figure 4. Porosity (A) and pores density (B) versus anodizing voltage for various electrolyte's temperatures

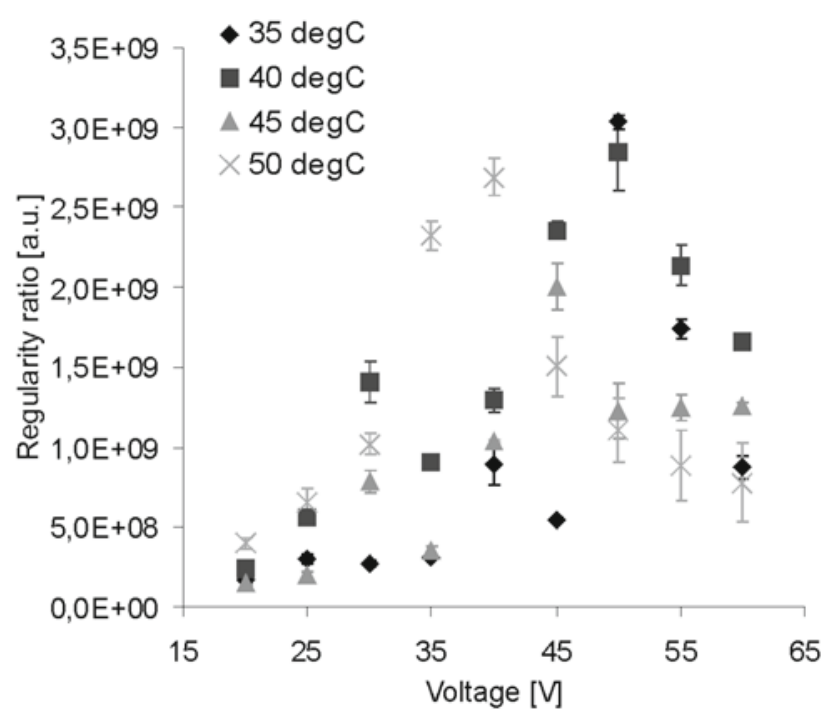

Figure 5. Fast Fourier transform based regularity ratio versus anodizing voltage for various electrolyte's temperatures 
theoretically calculated data, especially for anodizing the voltages close to the $40.0 \mathrm{~V}$.

A novel approach of the quantitative arrangement analysis based on the fast Fourier transforms radial averages is a fast and elegant method bringing the information about interpore distance distribution. Regularity ratio based on this method, reveals that the best interpore distance uniformity of the AAO formed in $0.3 \mathrm{M}$ oxalic acid is for the nanoporous arrays formed at voltages ranging from 40.0 to $50.0 \mathrm{~V}$.

\section{ACKNOWLEDGMENTS}

The research was financed by National Science Centre (Decision number: DEC-2012/05/B/ST8/02915).

\section{LITERATURE CITED}

1. Jani, A.M.M., Losic, D. \& Voelcker, N.H. (2013). Nanoporous anodic aluminium oxide: Advances in surface engineering and emerging applications. Prog. Mater. Sci. 58, 636-704. DOI: 10.1016/j.pmatsci.2013.01.002.

2. Zaraska, L., Sulka, G.D. \& Jaskuła, M. (2012). Fabrication of free-standing copper foils covered with highly-ordered copper nanowires arrays. Appl. Surf. Sci. 258, 7781-7785. DOI: $10.1016 /$ j.apsusc.2012.04.148.

3. Zaraska, L., Kurowska, E., Sulka, G.D. \& Jaskuła, M. (2012). Template-assisted fabrication of tin and antimony based nanowire arrays. Appl. Surf. Sci. 258, 9718-9722. DOI: 10.1016/j. apsusc.2012.06.018.

4. Feizi, E., Scott, K., Baxendale, M., Pal, C., Ray, A.K., Wang, W., Pang, Y. \& Hodgson, S.N.B. (2012). Synthesis and characterisation of nickel nanorods for cold cathode fluorescent lamps. Mat. Chem. Phys. 135, 832-836. DOI: 10.1016/j. matchemphys.2012.05.066.

5. Montero-Moreno, J.M., Belenguer, M., Sarret, M. \& Műller, C.M. (2009). Production of alumina templates suitable for electrodeposition of nanostructures using stepped techniques. Electrochim. Acta 54, 2529-2535. DOI: 10.1016/j. electacta.2008.03.067.

6. Liu, P., Singh, V.P., Jarro, C.A. \& Rajaputra, S. (2011). Cadmium sulfide nanowires for the window semiconductor layer in thin film CdS-CdTe solar cells. Nanotechnology 22, 145304. DOI: 10.1088/0957-4484/22/14/145304.

7. Márquez, F., Morant, C., López, V., Zamora, F., Campo, T. \& Elizalde, E. (2011). An alternative route for the synthesis of silicon nanowires via porous anodic alumina masks. Nanoscale Res. Lett. 6, 495. DOI: 10.1186/1556-276X-6-495.

8. Gomez, H., Riveros, G., Ramirez, D., Henriquez, R., Schrebler, R., Marotti, R. \& Dalchiele, E. (2012). Growth and characterization of $\mathrm{ZnO}$ nanowire arrays electrodeposited into anodic alumina templates in DMSO solution. J. Solid State Electrochem. 16, 197-204. DOI: 10.1007/s10008-011-1309-8.

9. Kokonou, M., Ioannou, G., Rebholz, C. \& Doumanidis, C.C. (2013). Polymeric nanowires and nanopillars fabricated by template wetting. J. Nanopart. Res. 15, 1552. DOI: 10.1007/ s11051-013-1552-2.

10. Lee, D.Y., Lee, D.H., Lee, S.G. \& Cho, K. (2012). Hierarchical gecko-inspired nanohairs with a high aspect ratio induced by nanoyielding. Soft Mater. 8, 4905-4910. DOI: 10.1039/C2SM07319F.

11. Yu, Y., Kant, K., Shapter, J.G., Addai-Mensah, J., Losic, D. (2012). Gold nanotube membranes have catalytic properties. Micropor. Mesopor. Mater. 153, 131-136. DOI: 10.1016/j. micromeso.2011.12.011.

12. Xu, X., Huang, J., Shao, M. \& Wang, P. (2012). Synthetic control of large-area, ordered Fe nanotubes and their nanotube-core/ alumina-sheath nanocables. Mat. Chem. Phys. 135, 6-9. DOI: 10.1016/j.matchemphys.2012.04.045.
13. Bocchetta, P., Santamaria, M. \& Di Quarto, F. (2013). One-step electrochemical synthesis and physicochemical characterization of CdSe nanotubes. Electrochim. Acta 88, 340-346. DOI: http://dx.doi.org/10.1016/j.electacta.2012.09.112.

14. Pitzschel, K., Bachmann, J., Montero-Moreno, J.M., Escrig, J., Görlitz, D. \& Nielsch, K. (2012). Reversal modes and magnetostatic interactions in $\mathrm{Fe}_{3} \mathrm{O}_{4} / \mathrm{ZrO}_{2} / \mathrm{Fe}_{3} \mathrm{O}_{4}$ multilayer nanotubes. Nanotechnology 23, 495718. DOI: 10.1088/09574484/23/49/495718.

15. Sarno, M., Tamburrano, A., Arurault, L., Fontorbes, S., Pantani, R., Datas, L., Ciambelli, P. \& Sarto, M.S. (2013). Electrical conductivity of carbon nanotubes grown inside a mesoporous anodic aluminum oxide membrane. Carbon 55, 10-22. DOI: 10.1016/j.carbon.2012.10.063.

16. Li, X., Lim, Y.F., Yao, K., Tay, F.E.H. \& Seah, K.H. (2013). Ferroelectric Poly(vinylidene fluoride) Homopolymer Nanotubes Derived from Solution in Anodic Alumina Membrane Template. Chem. Mater. 25, 524-529. DOI: 10.1021/ cm3028466.

17. Xu, X., Huang, J., Shao, M. \& Wang, P. (2012). Synthetic control of large-area, ordered $\mathrm{Fe}$ nanotubes and their nanotube-core/ alumina-sheath nanocables. Mat. Chem. Phys. 135, 6-9. DOI: 10.1016/j.matchemphys.2012.04.045.

18. Norek, M., Stępniowski, W.J., Zasada, D., Karczewski, K., Bystrzycki, J. \& Bojar, Z. (2012). $\mathrm{H}_{2}$ absorption at ambient conditions by anodized aluminum oxide (AAO) pattern-transferred Pd nanotubes occluded by Mg nanoparticles. Mat. Chem. Phys. 133, 376-382. DOI: 10.1016/j.matchemphys.2012.01.043.

19. Yu, D., Feng, Y., Zhu, Y., Zhang, X., Li, B., Liu, H. (2011). Template synthesis and characterization of molybdenum disulfide nanotubules. Mat. Res. Bull. 46, 1504-1509. DOI: 10.1016/j.materresbull.2011.04.018.

20. Valeev, R., Romanov, E., Beltukov, A., Mukhgalin, V., Roslyakov, I. \& Eliseev, A. (2012). Structure and luminescence characteristics of $\mathrm{ZnS}$ nanodot array in porous anodic aluminum oxide. Phys. Status Sol. C 9, 1462-1465. DOI: 10.1002/ pssc. 201100677.

21. Böhnert, T., Vega, V, Michel, A.K., Prida, V.M. \& Nielsch, K. (2013). Magneto-thermopower and magnetoresistance of single Co-Ni alloy nanowires. Appl. Phys. Lett. 103, 092407. DOI: $10.1063 / 1.4819949$.

22. Prida, V.M., García, J., Iglesias, L., Vega, V., Görlitz, D., Nielsch, K., Barriga-Castro, E.D., Mendoza-Reséndez, R., Ponce, A. \& Luna, C., (2013). Electroplating and magnetostructural characterization of multisegmented $\mathrm{Co}_{54} \mathrm{Ni}_{46} / \mathrm{Co}_{85} \mathrm{Ni}_{15}$ nanowires from single electrochemical bath in anodic alumina templates. Nanoscale Res. Lett. 8, 1-7. DOI: 10.1186/1556-276X-8-263.

23. Romero, V., Vega, V., García, J., Zierold, R., Nielsch, K., Prida, V.M., Hernando, B. \& Benavente, J. (2013). Changes in morphology and ionic transport induced by ALD $\mathrm{SiO}_{2}$ coating of nanoporous alumina membranes. ACS Appl. Mater. Interf. 5, 3556-3564. DOI: 10.1021/am400300r.

24. Yang, Z. \& Veinot, J.G.C. (2011). Size-controlled template synthesis of metal-free germanium nanowires. J. Mater. Chem. 21, 16505-16509. DOI: 10.1039/c1jm12460a.

25. Das, G. , Patra, N., Gopalakrishanan, A., Proietti Zaccaria, R., Toma, A, Thorat, S., Di Fabrizio, E., Diaspro, A. \& Salerno, M. (2012). Surface enhanced Raman scattering substrate based on gold-coated anodic porous alumina template. Microelectron. Eng. 97, 383-386. DOI: 10.1016/j.mee.2012.02.037.

26. Das, G., Patra, N., Gopalakrishnan, A., Zaccaria, R.P., Toma, A., Thorat, S., Di Fabrizio, E., Diaspro, A. \& Salerno, M. (2012). Fabrication of large-area ordered and reproducible nanostructures for SERS biosensor application. Analyst. 137, 1785-1792. DOI: 10.1039/c2an16022f.

27. Kurowska, E., Brzózka, A., Jarosz, M., Sulka, G.D. \& Jaskuła, M. (2013). Silver nanowire array sensor for sensitive and rapid detection of $\mathrm{H}_{2} \mathrm{O}_{2}$. Electrochim. Acta. 104, 439-447. DOI: 10.1016/j.electacta.2013.01.077 
28. Sulka, G.D., Hnida, K. \& Brzózka, A. (2013). pH sensors based on polypyrrole nanowire arrays. Electrochim. Acta. 104, 536-541. DOI: 10.1016/j.electacta.2012.12.064.

29. Salerno, M., Caneva-Soumetz, F., Pastorino, L., Patra, N., Diaspro, A. \& Ruggiero, C. (2013). Adhesion and Proliferation of Osteoblast-Like Cells on Anodic Porous Alumina Substrates With Different Morphology. IEEE Trans. Nanobiosci. 12, 106-111. DOI: 10.1109/TNB.2013.2257835.

30. Gultepe, E., Nagesha, D., Sridhar, S. \& Amiji, M. (2010). Nanoporous inorganic membranes or coatings for sustained drug delivery in implantable devices. Adv. Drug. Deliv. Rev. 62, 305-315. DOI: 10.1016/j.addr.2009.11.003.

31. Szuwarzyński, M., Zaraska, L., Zapotoczny, S. \& Sulka, G.D. (2013). Pulsatile releasing platform of nanocontainers equipped with thermally responsive polymeric nanovalves. Chem. Mater. 25, 514-520. DOI: 10.1021/cm303930y.

32. Ono, S. \& Masuko, N. (2003). Evaluation of pore diameter of anodic porous films formed on aluminum. Surf. Coat. Technol. 169-170, 139-142. DOI: 10.1016/S0257-8972(03)00197-X.

33. Sulka, G.D., Stroobants, S., Moshchalkov, V., Borghs, G. \& Celis, J.P. (2002). Synthesis of Well-Ordered Nanopores by Anodizing Aluminum Foils in Sulfuric Acid. J. Electrochem. Soc. 149, D97-D103. DOI: 10.1149/1.1481527.

34. Sulka, G.D. \& Stępniowski, W.J. (2009). Structural features of self-organized nanopore arrays formed by anodization of aluminum in oxalic acid at relatively high temperatures. Electrochim. Acta 54, 3683-3691. DOI: 10.1016/j.electacta.2009.01.046.

35. Stępniowski, W.J., Norek, M., Michalska-Domańska, M. \& Bojar, Z. (2013). Ultra-small nanopores obtained by self-organized anodization of aluminum in oxalic acid at low voltages. Mater. Lett. 111, 20-23. DOI: 10.1016/j.matlet.2013.08.059.

36. Stępniowski, W.J., Nowak-Stępniowska, A. \& Bojar, Z. (2013). Quantitative arrangement analysis of anodic alumina formed by short anodizations in oxalic acid. Mater. Character. 78, 79-86. DOI: 10.1016/j.matchar.2013.01.013.

37. Zaraska, L., Sulka, G.D. \& Jaskuła, M. (2010). The effect of n-alcohols on porous anodic alumina formed by self-organized two-step anodizing of aluminum in phosphoric acid. Surf. Coat. Technol. 204, 1729-1737. DOI: 10.1016/j.surfcoat.2009.10.051.

38. Ono, S., Saito, M. \& Asoh, H. (2005). Self-ordering of anodic porous alumina formed in organic acid electrolytes. Electrochim. Acta. 51, 827-833. DOI: 10.1016/j.electacta.2005.05.058.

39. Pashchanka, M. \& Schneider, J.J. (2013). Experimental validation of the novel theory explaining self-organization in porous anodic alumina films. Phys. Chem. Chem. Phys. 15, 7070-7074. DOI: 10.1039/c3cp50805f.

40. Kikuchi, T., Yamamoto, T. \& Suzuki, R.O. (2013). Growth behavior of anodic porous alumina formed in malic acid solution. Appl. Surf. Sci. 284, 907-913. DOI: 10.1016/j. apsusc.2013.08.044.

41. Patra, N., Salerno, M., Losso, R., Cingolani, R. (2009). Use of unconventional organic acids as anodization electrolytes for fabrication of porous alumina. 2009 9th IEEE Conference on Nanotechnology, IEEE NANO 2009, 26-30 July 2009 (pp. 567-570). Genoa, Italy. WSxM. http://www.nanotec.es

42. Horcas, I., Fernández, R., Gómez-Rodríguez, J.M., Colchero, J., Gómez-Herrero, J. \& Baro, A.M. (2007). WSXM: a software for scanning probe microscopy and a tool for nanotechnology. Rev. Sci. Instrum. 78, 013705. DOI: 10.1063/1.2432410.

43. Sulka. G.D. \& Parkoła. K.G. (2007). Temperature influence on well-ordered nanopore structures grown by anodization of aluminium in sulphuric acid. Electrochim. Acta. 52, 1880-1888. DOI: 10.1016/j.electacta.2006.07.053.

44. Zaraska. L., Stępniowski. W.J., Ciepiela. E. \& Sulka. G.D. (2013). The effect of anodizing temperature on structural features and hexagonal arrangement of nanopores in alumina synthesized by two-step anodizing in oxalic acid. Thin Solid Films 534, 155-161. DOI: 10.1016/j.tsf.2013.02.056.

45. Pashchanka, M. \& Schneider, J.J. (2011). Origin of self-organisation in porous anodic alumina films derived from analogy with Rayleigh-Bénard convection cells. J. Mater. Chem. 21, 18761-18767. DOI: 10.1039/c1jm13898g.

46. Nielsch, K, Choi, J., Schwirn, K., Wehrspohn, R.B. \& Gösele, U. (2002). Self-ordering Regimes of Porous Alumina: The 10\% Porosity Rule. Nano Lett. 2, 677-680. DOI: 10.1021/ nl025537k. 